\title{
Die Erwerbstätigkeit im Alter steigt - die Beschäftigungslosigkeit auch
}

\author{
Martin Brussig, Matthias Knuth, Sascha Wojtkowski
}

Vor gut zwei Jahren diskutierten wir in den WSI-Mitteilungen die damals geplante Heraufsetzung des gesetzlichen Renteneintrittsalters von 65 auf 67 Jahre. $^{1}$ Der Gesetzgeber hat diese Änderungen mittlerweile umgesetzt, sie werden ihre unmittelbaren Auswirkungen jedoch erst ab 2012 zeigen. Einstweilen entfalten die vorausgegangen, auf Zurückdrängung vorzeitiger Verrentungen gerichteten Rentenreformen ihre Wirkung. Die Erwerbstätigkeit Älterer nimmt zu, das durchschnittliche Rentenzugangsalter steigt, die Frühverrentung geht zurück. Kaum eine Reform scheint so effektiv und geräuschlos zu wirken wie diese. Darüber könnte übersehen werden, dass diese Reform bereits jetzt zu erkennbar wachsenden sozialen Ungleichheiten führt.

\section{Einleitung}

Ebenso wie andere Länder der Europäischen Union (EU) hat Deutschland den wirtschaftlichen Strukturwandel jahrelang unter anderem durch die vorzeitige Stilllegung des Arbeitskräftepotenzials von Älteren bewältigt. Der Strukturbruch, der auf die deutsche Einigung folgte, führte zur Vertiefung und Verlängerung dieses Politikmusters. Vor diesem Hintergrund erforderte das von den Mitgliedsländern der Europäischen Union gesetzte beschäftigungspolitische Ziel, „mehr Menschen in Arbeit zu bringen und zu halten" (EU 2005, L 205/21) und die Erwerbstätigenquote der Älteren zwischen 55 und 64 Jahren bis 2010 in jedem Mitgliedsland auf $50 \%$ zu erhöhen (EU 2001), einen besonders deutlichen Richtungswechsel in der deutschen Arbeitsmarkt- und Rentenpolitik. Dies gilt erst recht, wenn auch die qualitativen beschäftigungspolitischen Ziele der EU erfüllt werden sollen. Hierzu zählt, „Arbeitsplatzqualität, einschließlich Arbeitsentgelt und Sozialleistungen, Arbeitsbedingungen, Beschäftigungssicherheit (und) Zugang zum lebenslangen Lernen“" (EU 2005, L 205/24) zu gewährleisten. Bislang haben die Reformen in der Arbeitsmarkt- und Rentenpolitik vor allem Anreize für einen vorzeitigen Rentenbeginn und einen frühzeitigen Rückzug vom Arbeitsmarkt abgebaut, aber vergleichsweise wenig zur aktiven Beschäftigungsförderung Älterer beigetragen. Als bisher einziges Land in der EU hat Deutschland die Anhebung der Altersgrenze für die Regelaltersrente (ab 67 Jahre) beschlossen.

Sowohl die Reformen als auch ihre Auswirkungen werden kontrovers beurteilt:
Während die einen auf einen „unvollständigen Paradigmenwechsel“ (Eichhorst 2006) hinweisen und darauf, dass „die Weichen $(\ldots)$ noch nicht in die gewünschte Richtung [führen]" (Eichhorst/Sproß 2005), erinnern andere daran, dass die Anhebung der Regelaltersgrenze nicht in eine Zeit passt, in der bereits die bisherige Altersgrenze von 65 Jahren nur im Ausnahmefall aus einer Erwerbstätigkeit heraus erreicht wird (Ebert et al. 2006). Nicht minder kontrovers werden die Entwicklung der Alterserwerbstätigkeit und damit die Wirkungen der Reformen beurteilt: Bellmann et al. (2006) sehen Deutschland immer noch deutlich unterhalb des EU-Durchschnitts, während die Bundesagentur für Arbeit (BA) schon ein Jahr nach Verabschiedung der Reform mitteilte, dass die Erwerbstätigenquote Älterer die 50 \%-Marke erreicht habe und damit über dem EU-Durchschnitt liege (BA 2007; Eichhorst 2008).

Angesichts solch heterogener Einschätzungen und Angaben ist es notwendig, über ein Berichtssystem zur Erwerbstätigkeit im Alter und zum Rentenübergang zu verfügen, das aktuell und differenziert den Altersübergang darstellen kann. Entsprechende Daten stellt das Institut für Arbeit und Qualifikation der Universität Duisburg-Essen seit einigen Jahren bereit. In diesem Beitrag stellen wir Ergebnisse aus unseren Datenanalysen vor. ${ }^{2}$

\section{Stark zunehmende Alterserwerbstätigkeit}

Die Erwerbstätigkeit der 55- bis 64-Jährigen $^{3}$ in Deutschland ist in den letzten Jahren von $38,0 \%$ auf $48,1 \%$ gestiegen, wobei
1 Vgl. Brussig/Knuth 2006

2 Unter dem Titel "Altersübergangsmonitor " fördert die Hans-Böckler-Stiftung seit 2004 dieses Berichtssystem. Seit 2006 ist auch das Forschungsnetzwerk Alterssicherung der Deutschen Rentenversicherung Bund an seiner Finanzierung beteiligt. Die bisher erschienen Ausgaben des Altersübergangs-Reports können heruntergeladen werden unter: http://www.iaq.uni-due.de/auemreport/

3 In international vergleichenden Studien wird die Erwerbstätigenquote überwiegend nach dem Labour-Force-Konzept der International Labour Organisation (ILO) gemessen, das auch wir hier verwenden. Als erwerbstätig gilt, wer im Berichtszeitraum nach eigener Angabe mindestens eine Stunde pro Woche eine entgeltliche Tätigkeit ausgeübt hat, unabhängig von der Stellung als Arbeitnehmer, Selbstständiger oder mithelfender Familienangehöriger und unabhängig vom etwaigen gleichzeitigen Bezug von Sozialleistungen. In Deutschland zählen auch Arbeitsgelegenheiten ( „EinEuro-Jobs“) als Erwerbstätigkeit. Auch wer sich formal in einem Arbeitsverhältnis befindet, das im Berichtszeitraum vorübergehend nicht ausgeübt wurde, gilt als erwerbstätig (Personen in der Freistellungsphase der Altersteilzeit, Frauen im Mutterschutz, Personen in Elternzeit mit Rückkehrrecht und Personen, die urlaubs- oder krankheitsbedingt ihrer Arbeit nicht nachgehen).

Martin Brussig, Dr., wissenschaftlicher Mitarbeiter am Institut Arbeit und Qualifikation (IAQ) der Universität Duisburg-Essen. Arbeitsschwerpunkte: Erwerbstätigkeit und demografischer Wandel; Arbeitsmarktpolitik. e-mail: martin.brussig@uni-due.de Matthias Knuth, Prof. Dr., Abteilungsleiter am IAQ. Arbeitsschwerpunkte: Arbeits- und Sozialpolitik, Erwerbssysteme. e-mail: matthias.knuth@uni-due.de Sascha Wojtkowski, Dipl.-Soz., Wiss., wissenschaftlicher Mitarbeiter am IAQ. Arbeitsschwerpunkte: Arbeits- und Sozialpolitik, Mitbestimmung. e-mail: sascha.wojtkowski@uni-due.de 
die Zunahme nach 2001einsetzte. Der aktuelle deutsche Wert ist zwar noch weit entfernt von europäischen Spitzenreitern der Alterserwerbstätigkeit, wie Dänemark oder Schweden - jeweils über $60 \%-$, doch in keinem anderen Land der EU 15 war die Zunahme der Alterserwerbstätigkeit so stark wie in Deutschland. Dieses ist besonders bemerkenswert vor dem Hintergrund, dass die Erwerbstätigenquote der 15-54Jährigen im gleichen Zeitraum allenfalls konjunkturbedingte Schwankungen zeigt (Abbildung 1).

Für diesen starken Anstieg gibt es drei Gründe: Erstens wurden in Deutschland im Jahr 2005 Details im Erhebungsverfahren und bei der Berechnung der Erwerbstätigenquote umgestellt, die für einen kräftigen Einmaleffekt im Jahr 2005 sorgten. Allerdings betont das hierfür verantwortliche Statistische Bundesamt, dass diese Anpassungen die tatsächliche Erwerbsbeteiligung besser widerspiegelt (Deutscher Bundestag 2007, S. 3). Demzufolge wird nicht aktuell die Erwerbsbeteiligung der Älteren überschätzt, vielmehr wurde sie vor 2005 unterschätzt. Es waren aber diese wie sich im Nachhinein herausstellt: unzureichenden - Datengrundlagen, die auch zur Begründung weitreichender Arbeitsmarktreformen genutzt wurden.

Der zweite Grund für die besonders starke Zunahme der Alterserwerbstätigkeit ist demografischer Natur. Der demografische Wandel wird gemeinhin nur als zunehmendes Durchschnittsalter der (Erwerbs-) Bevölkerung und in Form höherer Anteile Älterer in der Gesellschaft und Arbeitswelt wahrgenommen. Tatsächlich aber wird das durch die Zielsetzungen der EU definierte Altersfenster zwischen dem 55 . und dem 64. Lebensjahr derzeit von Geburtsjahrgängen mit außerordentlich unterschiedlichen Jahrgangsstärken durchlaufen. Nachdem die stark besetzten Geburtsjahrgänge um 1941 inzwischen das 65. Lebensjahr überschritten haben, gibt es vorübergehend weniger Personen als vorher, die sich im kritischen letzten Fünftel ihres Erwerbsalters befinden. Diese Entwicklung trifft zusammen mit einer günstigen Konjunktur, was insgesamt entlastend für die Altersbeschäftigung wirkt. Nachdem ferner der Jahrgang 1945 als der am schwächsten besetzte das 60. Lebensjahr überschritten hat und nun wieder etwas geburtenstärkere Jahrgänge das kritische Altersfenster von 55 bis 64 erreichen, wird dieses nunmehr von ,jungen Alten“

Abb. 1: Erwerbstätigenquoten der Altersgruppen 15-54 Jahre sowie 55-64 Jahre in Deutschland, 1996 - 2006* - in \% -

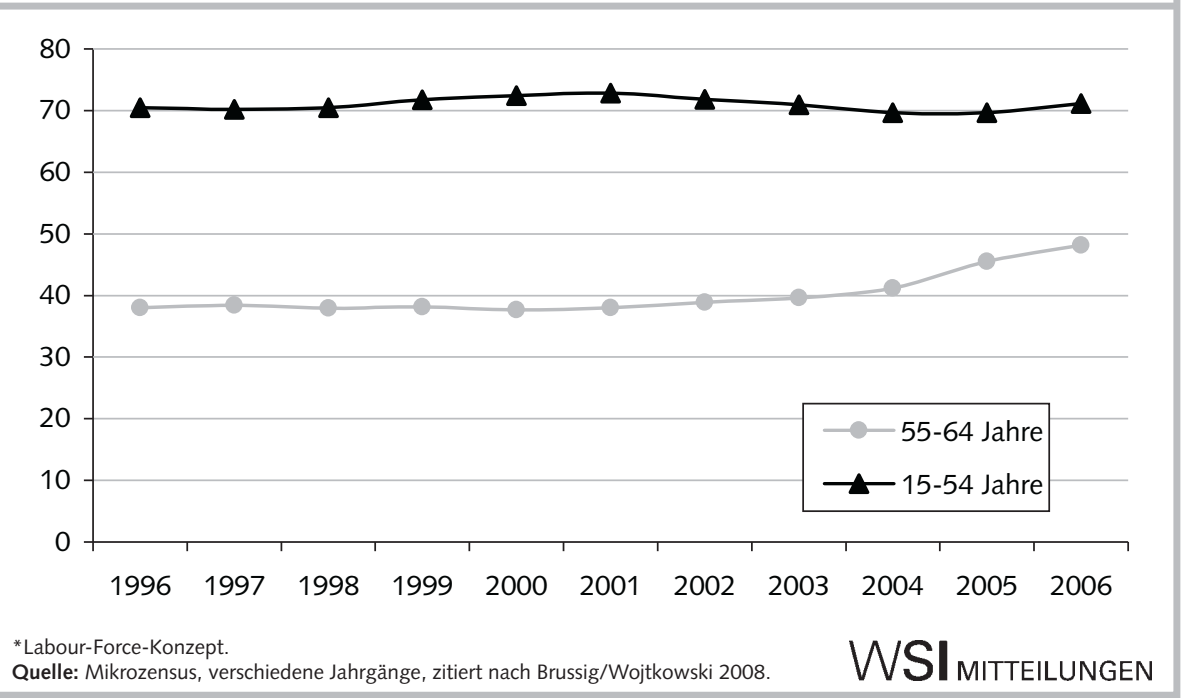

\section{Abb. 2: Altersspezifische Erwerbstätigenquoten nach Geschlecht, 1996, 2001, 2006* - in \% -}
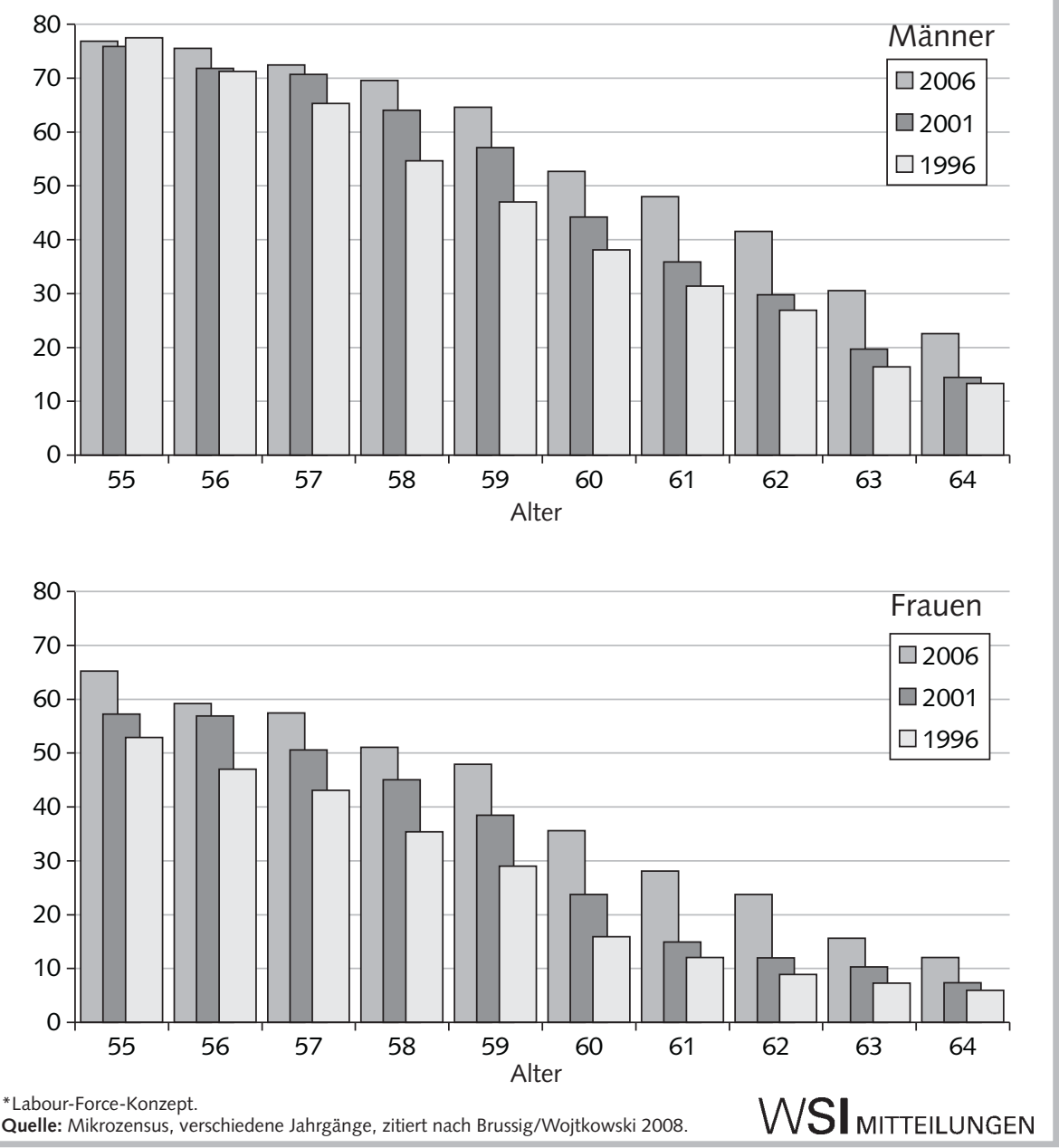

unter 60 dominiert. Bezogen auf die $\mathrm{Al}-$ tersgruppe 55-64 insgesamt erhöht das die Wahrscheinlichkeit der Erwerbstätigkeit. Man kann den daraus resultierenden Effekt auf die Erwerbstätigenquote der Älte- ren schätzen, indem man die Altersstruktur von 2006 mit den altersspezifischen Erwerbstätigenquoten des Jahres 2001 kombiniert - einem Jahr, in dem das interessierende Altersfenster noch von ,alten Alten“ 


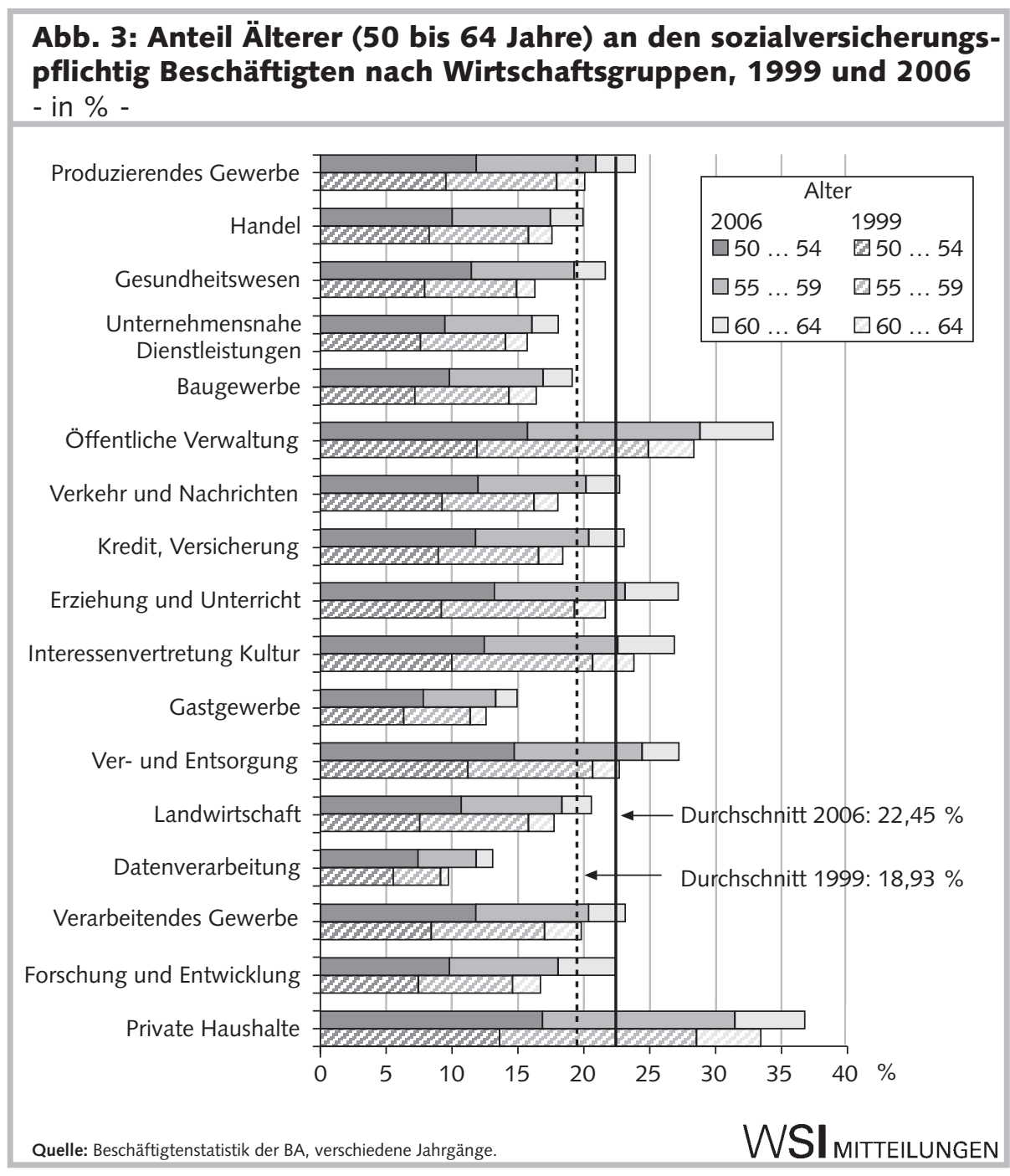

dominiert wurde. Demzufolge wären ca. $20 \%$ der beobachteten Zunahme der Erwerbstätigenquote der 55-64-Jährigen auch dann eingetreten, wenn sich an den altersspezifischen Wahrscheinlichkeiten für Erwerbstätigkeit nichts geändert hätte (Brussig/Wojtkowski 2008). Dieser Anteil der Steigerung ist folglich auf den demografischen Struktureffekt zurückzuführen. ${ }^{4}$ „Demografie“ ist eben nicht immer nur Bedrohung und heißt auch mehr als „Alterung“ - im kritischen Übergangsalter schafft sie derzeit und noch bis ca. 2023 Entlastung, nämlich bis die Spitze des Baby-Booms 1963/1964 das 60. Lebensjahr überschritten hat und dadurch das Durchschnittsalter in der Gruppe der 55-64Jährigen wieder über 60 steigen lässt.

Wenn $20 \%$ des Anstiegs der Alterserwerbsquote auf den Altersstruktureffekt zurückzuführen sind, dann stellt zunehmende Erwerbsbeteiligung den dritten und wichtigsten Grund dar. Bei Männern hat die Erwerbstätigkeit insbesondere im Alter zwischen 58 und 62 Jahren zugenommen

(Abbildung 2). Diese Altersspanne war in den 1980er und 1990er Jahren das biografische Zeitfenster der Frühausgliederung, die heute deutlich erschwert ist. Bei Frauen hat die Erwerbsbeteiligung in jeder Altersstufe zugenommen, weil Geburtskohorten mit stärkerer Erwerbsorientierung in das betrachtete Altersfenster hineinwachsen. Nach wie vor auffällig ist bei den Frauen der Sprung zwischen dem 59. und dem 60. Lebensjahr: Einerseits muss auch die Frauenaltersrente zunehmend mit Rentenabschlägen erkauft werden (Abschnitt 4), andererseits jedoch ist beitragspflichtige Beschäftigung in der zweiten Lebenshälfte die jetzt auch von einem größeren Anteil älterer Frauen erfüllte Voraussetzung, um überhaupt diese Rentenart in Anspruch nehmen zu können.

Die Erwerbsintegration im Alter hängt sehr stark von der Qualifikation ab (ausführlich Brussig/Knuth 2006): Zwei Drittel der Älteren mit Fachhochschul- oder Hochschulabschluss $(68,7 \%)$ waren im Jahr 2006 noch beschäftigt, aber nur ein Drittel
(35,5 \%) der Älteren ohne beruflichen Abschluss. Im europäischen Vergleich ist gerade in Deutschland die Erwerbsintegration im Alter besonders stark von der Qualifikation abhängig (Bosch/Schief 2005). Die Beschäftigungsfähigkeit von Geringqualifizierten wird wegen lebenslang ungünstiger Arbeitsbedingungen und Erwerbsverläufe im Alter prekär; gerade ihnen fehlen die von der EU-Beschäftigungsstrategie geforderten Zugänge zum lebenslangen Lernen.

Die Zunahme der Altersbeschäftigung ist nicht auf einzelne Branchen beschränkt (Abbildung 3). ${ }^{5}$ Zwar gibt es erhebliche Unterschiede zwischen Branchen: Im Baugewerbe, im Gastgewerbe und in der kleinen Branche „Datenverarbeitung “ stellen Ältere nur einen geringen Anteil an den Beschäftigten, während ihr Anteil im Öffentlichen Dienst und benachbarten Bereichen (Erziehung und Unterricht, Interessenvertretung und Kultur) überproportional ist. Daran hat sich zwischen 1999 und 2006 auch wenig geändert. Doch sowohl in Branchen mit einem niedrigen als auch mit einem hohen Anteil Älterer ist der Anteil der älteren Beschäftigten jeweils gestiegen. Gleiches gilt in wachsenden wie auch in schrumpfenden Beschäftigungssektoren.

\section{Wachsende Beschäftigungslosigkeit unter Älteren}

Dass die Alterserwerbstätigkeit zugenommen hat, bedeutet nicht, dass weniger Ältere beschäftigungslos wären - zunehmende Erwerbsbeteiligung kann einen gleichzeitigen Anstieg von Erwerbstätigkeit und Erwerbslosigkeit beinhalten. Auch hier sind -

\footnotetext{
4 Eine alternative Simulation von Kistler et al. (2007) kommt zu einem deutlich höheren demografischen Effekt. Allerdings werden dort Beschäftigungsquoten der BA zugrundegelegt, und die Gruppe der 55- bis 64-Jährigen wird nicht in zehn Teilgruppen (für jeden Geburtsjahrgang eine), sondern nur in zwei Teilgruppen (55- bis 59-Jährige und 60- bis 64-Jährige) zerlegt. Im Beobachtungszeitraum wechselte aber der extrem schwach besetzte Jahrgang 1945 von der einen Teilgruppe in die andere, was einen Effekt auf das Ergebnis haben dürfte.

5 Zu beachten ist, dass sich diese Darstellung - im Unterschied zu den vorangegangenen Betrachtungen - einerseits auf sozialversicherungspflichtig Beschäftigte beschränkt, andererseits auch die Altersgruppe 50-54-Jähriger umfasst.
} 
wie bei der Erwerbstätigkeit - die statistischen Grundlagen zur Bestimmung von Arbeitslosigkeit bzw. Beschäftigungslosigkeit zu beachten. Zwar geht die Zahl der jahresdurchschnittlich bei der BA registrierten älteren Arbeitslosen ab 50 Jahren seit ca. 1997 zurück (1,35 Mio.) und liegt seit 2000 beständig unter 1,2 Mio. Abgenommen hat vor allem die Zahl der 58jährigen (und älter) registrierten Arbeitslosen, während die der 50- bis 57-jährigen ihren Höhepunkt erst 2005 erreicht hat (ca. 1,0 Mio., vgl. hierzu auch Brussig/Wojtkowski 2007a, 2007b).

Die Abnahme der Zahl und noch stärker des Anteils der registrierten Arbeitslosen ab 58 Jahren ist jedoch nicht in erster Linie auf eine bessere Erwerbsintegration (oder stärkere Aufnahme in arbeitsmarktpolitische Programme) zurückzuführen, sondern auf die Möglichkeit des „Leistungsbezugs unter erleichterten Voraussetzungen“: Arbeitslose ab 58 Jahren konnten bis Ende 2007 weiterhin Arbeitslosengeld, Arbeitslosenhilfe (bis 2004) bzw. Arbeitslosengeld II ( $a b$ 2005) beziehen, ohne dem Arbeitsmarkt zur Verfügung zu stehen, wenn sie sich verpflichteten, zum frühestmöglichen Zeitpunkt abschlagsfrei eine Altersrente zu beantragen. Sie werden dann nicht mehr als arbeitslos registriert, sondern zählen als ,nicht arbeitslose Leistungsempfänger“. Die Entwicklung bis 2003 ist im Einzelnen dargestellt in Brussig/ Knuth 2006. Im Jahre 2004, d.h. unmittelbar vor der Einführung der Grundsicherung für Arbeitsuchende (Hartz IV), waren fast zwei Drittel der beschäftigungslosen Leistungsempfänger ab 58 Jahren nicht als arbeitslos registriert. Die Einführung des SGB II hat die Möglichkeiten zur Fortschreibung der Statistik beeinträchtigt. Denn für 2005 und 2006 hat die Bundesagentur für Arbeit nur noch die Empfänger von Arbeitslosengeld im „erleichterten Leistungsbezug" ausgewiesen, aber nicht mehr die Bezieher von „Arbeitslosengeld II“, die unter diese Bestimmung fielen. ${ }^{6}$ Seit 2008 verschwinden Arbeitslosengeld-II-Beziehende mit Vollendung des 59. Lebensjahres automatisch aus der Arbeitslosenstatistik, wenn ihnen in den vorausgegangen zwölf Monaten keine sozialversicherungspflichtige Beschäftigung angeboten worden ist ( $\$ 53$ a SGB II). Diese Bestimmung kommt einem faktischen Ausschluss aus Vermittlung und Beschäftigungsförderung ab dem 58. Lebensjahr gleich und steht in diametralem Widerspruch zum Anspruch der Bundesregie-

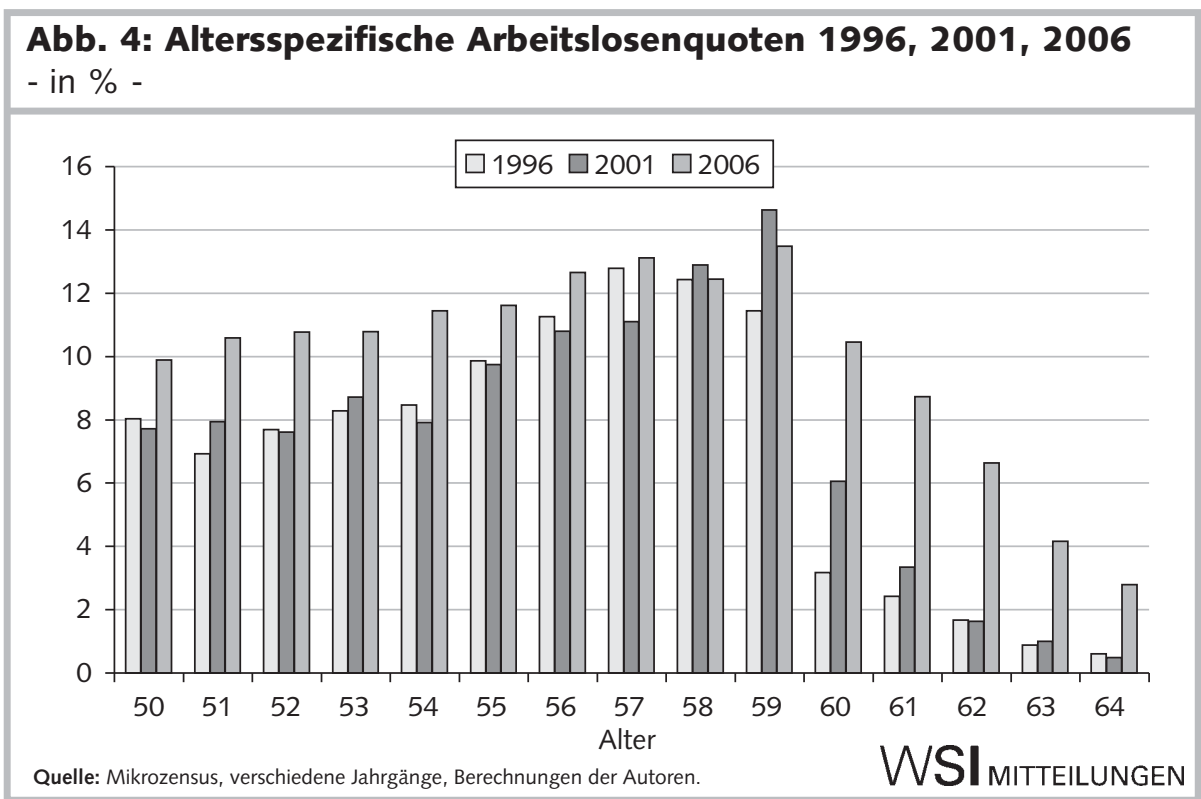

rung, die Beschäftigungsaufnahme Älterer zu fördern.

Die Beschäftigungslosigkeit Älterer hat auch deshalb zugenommen, weil ein erheblicher Teil der Arbeitslosen nicht wie früher zum frühestmöglichen Zeitpunkt mit 60 Jahren in Altersrente geht, sondern den Rentenbeginn aufschiebt, um die Abschläge bei vorzeitigem Rentenzugang zu verringern. In den letzten Jahren hat sich Arbeitslosigkeit jenseits von 60 Jahren überhaupt erst in einem nennenswerten Umfang herausgebildet, wie die Selbstauskünfte von Befragten im Mikrozensus zeigen (Abbildung 4).

Da viele nun längere Zeit als früher in Arbeitslosigkeit ihren Rentenbeginn abwarten, ergibt sich das nur scheinbar widersprüchliche Ergebnis, dass Erwerbstätigkeit und Beschäftigungslosigkeit unter den Älteren zunehmen.

\section{Weiterer Anstieg des Rentenzugangsalters, aber zunehmende Differenzierung}

Der Anstieg des durchschnittlichen Rentenzugangsalters, der bereits geraume Zeit beobachtet werden kann (Brussig/Knuth 2006), hat sich in der jüngsten Vergangenheit verlangsamt. Das durchschnittliche Rentenzugangsalter (nur Altersrenten) betrug 2005 und 2006 jeweils 63,4 Jahre $^{7}$ und ist damit seit 1999 um fast ein Jahr angestiegen. Doch auch diese Werte werden von demografischen Unregelmäßigkeiten beeinflusst. In den jährlichen Zugängen in Altersrenten, aus denen der Durchschnitt gebildet wird, treffen die "Langläufer" einer Geburtskohorte mit den „Frührentnern“ aus den vier nächstjüngeren Geburtskohorten zusammen. Wenn die Kohorte, die das 65. Lebensjahr erreicht, stärker besetzt ist als die der Jüngeren, dann wirkt das steigernd auf das durchschnittliche Zugangsalter; bei umgekehrtem Größenverhältnis der Kohorten tritt der gegenteilige Effekt ein. Nachdem der Jahrgang 1941, der stärker besetzt ist als die nachfolgenden Jahrgänge, die Regelaltersgrenze passiert hat, kehrt sich der Altersstruktureffekt, der bis 2005 steigernd auf das durchschnittliche Rentenzugangsalter gewirkt hat, vorübergehend um. Die zu erwartende Stagnation

6 Aus der IAB-Querschnittsbefragung ist bekannt, dass die Inanspruchnahme des erleichterten Leistungsbezuges von ALG-II-Empfängern deutlich niedriger war als unter Arbeitslosengeldempfängern; die Quote betrug weniger als $50 \%$. Hauptgrund ist, dass ALG-II-Empfänger, die vor der Einführung des SGB II Sozialhilfe bezogen haben, keine Möglichkeit zum erleichterten Leistungsbezug hatten (diese bestand nur im Rechtskreis des SGB III, also für Arbeitslosengeld- und -hilfebezieher) und in der Zeit ihres ALG-II-Bezuges - in der ihnen der erleichterte Leistungsbezug offen stand - diesen nicht genutzt haben (Brussig/Wübbeke 2008).

7 Häufig zitierte niedrigere Werte beziehen $\mathrm{Er}$ werbsminderungsrenten ein, die aber wenig mit dem Übergang von Erwerbstätigkeit in Rente am Ende des Erwerbslebens zu tun haben. Das Durchschnittsalter beim Zugang in Erwerbsminderungsrenten betrug 2006 50,1 Jahre und beim Zugang in alle Versichertenrenten (Altersrenten und Erwerbsminderungsrenten) 61,2 Jahre (Deutsche Rentenversicherung Bund 2007, S. 99). 


\section{Abb. 5: Entwicklung des durchschnittlichen Zugangsalters in Altersrenten, 1996-2007}

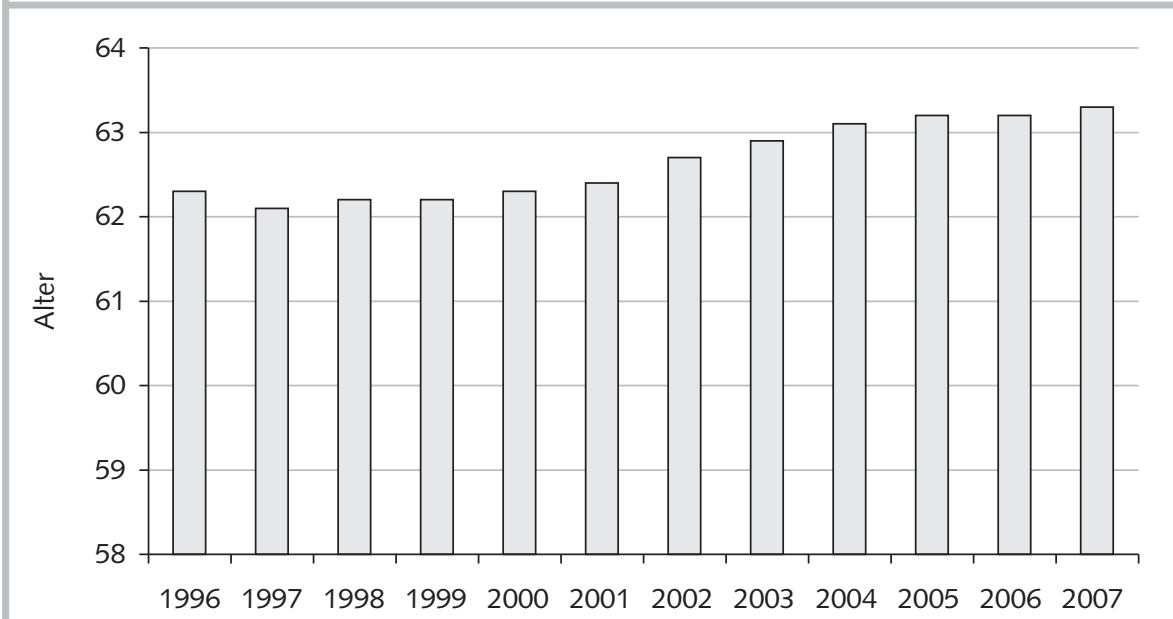

Quelle: Rentenversicherung in Zeitreihen 2007, S. 99; Deutsche Rentenversicherung in Zahlen 2008, S. 70

WSI

Abb. 6: Anteil der Altersrentenzugänge mit Abschlägen nach Geschlecht und Gebiet*, 2003-2005 - in \% -

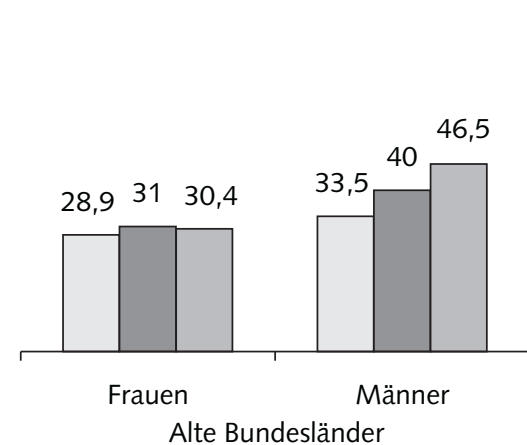

$$
63,7071,5
$$

$\square$ Rentenzugang $2003 \square$ Rentenzugang $2004 \square$ Rentenzugang 2005

*Anteil der Rentenzugänge mit Abschlägen an allen Rentenzugängen in \%. Quelle: FDZ-RV SUFRTZN03/04/05XVSBB, zitiert nach: Altersübergangsreport 200701, S. 5

dieses Wertes in den nächsten Jahren ist deshalb kein Anlass zur Beunruhigung. Dass es unabhängig von den Auswirkungen demografischer Unregelmäßigkeiten zu Verhaltensänderungen im Sinne einer Verlagerung der Renteneintritte in ein höheres Lebensalter gekommen ist, wurde mit drei unterschiedlichen Ansätzen demonstriert: Durch die Berechnung von „Rentenzugangsquotienten“ der Bevölkerung nach Einzelalter (Büttner/Knuth 2004; Brussig/ Wojtkowski 2006), durch die Berechnung eines auf die Altersstruktur standardisierten durchschnittlichen Rentenzugangsalters (Hoffmann 2007, S. 304) sowie durch die Bildung von Rentenbestandsquoten von Versichertenjahrgängen nach Einzelalter (ebd. S. 305). Lediglich die - methodisch am wenigsten problematische- nahezu halbiert hatte (von $40 \%$ auf ca. 23 $\%)$, wächst er seitdem wieder geringfügig an. Ähnlich verlief die Entwicklung der Zugänge in Altersrente wegen Arbeitslosigkeit: Nach einem langen Rückgang von $17 \%$ (1996) auf $5 \%$ (2003) ist der Anteil dieser Rentenart an allen Rentenzugängen in den Folgejahren bis 2005 wieder leicht gestiegen: auf 6,6\%. Dieser Anstieg ist gering, doch er fällt zeitlich zusammen mit der vierten Stufe der Hartz-Reformen, durch die unter anderem die maximale Bezugsdauer an Arbeitslosengeld für Ältere gekürzt wurde und mit der die Arbeitslosenhilfe durch das Arbeitslosengeld II abgelöst wurde, das in der Regel geringer bemessen wird und strengen Bedürftigkeitsprüfungen unterliegt. Es liegt nahe zu vermuten, dass eine nennenswerte Anzahl älterer Arbeitsloser mangels Anspruch auf Arbeitslosengeld II in die Altersrente „geflüchtet“ ist, wodurch die Bemühungen, das faktische Renteneintrittsalter zu steigern, für ältere Arbeitslose konterkariert werden (Brussig/Wojtkowski 2006).

Entwicklung und Höhe der Abschläge beim vorzeitigen Rentenbeginn geben ebenfalls Aufschluss über die soziale Differenzierung im Rentenzugang. Abschläge vom Rentenanspruch werden fällig, wenn der Rentenantrag vor Erreichen einer abschlagsfreien Altersgrenze gestellt wird. Pro Monat des vorgezogenen Rentenbeginns vermindert sich der Rentenanspruch um $0,3 \%$. Da der frühestmögliche Beginn einer Altersrente bei 60 Jahren und der abschlagsfreie Beginn bei 65 Jahren liegt, kann es zu Abschlägen von bis zu $18 \%$ kommen. Damit soll die längere Rentenbezugszeit versicherungsmathematisch ausgeglichen werden (Börsch-Supan 2004; Pimpertz 2004; Ohsmann et al. 2004).

Empirische Analysen zeigen übereinstimmend, dass mit Beginn der Altersgrenzenanhebung (1997) immer mehr Altersruhegeld-Empfänger Abschläge in Kauf nehmen müssen. Seit demselben Zeitpunkt steigt auch die durchschnittliche Höhe der Abschläge kontinuierlich an (Kaldybajewa 2004; Brussig 2007, siehe auch Abbildung 6). $42 \%$ aller Altersrentenzugänge des Jahres 2005 waren von Abschlägen betroffen. Im Durchschnitt erfolgen die Renteneintritte um mehr als drei Jahre vorzeitig (38,9 Monate im Jahr 2005 gegenüber 34,8 Monaten 2003). Wie in Abbildung 6 erkennbar, sind in Ostdeutschland deutlich mehr Ruhegeldempfänger von Abschlägen betroffen; hier gibt es auch nur geringe Ge- 
schlechterunterschiede. In Westdeutschland hingegen sind Männer wegen ihrer stärkeren Erwerbsintegration, die erst die Möglichkeit auf einen vorzeitigen Rentenbeginn eröffnet, stärker betroffen als Frauen; bei westdeutschen Frauen wiederum ist für die letzten Jahre keine weitere Zunahme der Betroffenheit mehr festzustellen.

Einen erheblichen Einfluss darauf, ob ein Rentenbeginn vorzeitig erfolgt, hat die Erwerbsbiografie unmittelbar vor Rentenbeginn. In typisierender Betrachtung lassen sich drei Konstellationen des Altersübergangs unterscheiden, die zusammen fast zwei Drittel (2005: 62,0 \%) aller Altersrentenzugänge ausmachen:

(1) In einer ersten Konstellation befinden sich Personen, die jeweils zum 31.12. in den drei Jahren vor Rentenbeginn versicherungspflichtig beschäftigt waren („durchgängig Beschäftigte“). Hierunter fallen auch Altersteilzeitbeschäftigte. Aus dieser Gruppe kamen 2005 30,8 \% aller neuen Altersrentner.

(2) In einer zweiten Konstellation sind Personen erfasst, für die in den letzten drei Jahren durchgängig ein Leistungsbezug nach SGB III (Arbeitslosengeld/-hilfe) oder die Zahlung von Krankengeld bzw. ähnlicher Leistungen oder Anrechnungszeiten ${ }^{8}$ bei der Rentenversicherung registriert ist. ${ }^{9}$ Ebenfalls enthalten sind geringfügig Beschäftigte. Diese Formen des Erwerbsstatus sind charakteristisch für Personen, die wegen Arbeitslosigkeit oder Krankheit nicht oder mit ihrer geringfügigen Beschäftigung nur wenig erwerbstätig sein konnten oder wollten („langer prekärer Altersübergang“). Jeder fünfte Neuzugang 2005 gehörte dieser Gruppe an (19,5\%), in den neuen Bundesländern macht diese Gruppe ca. $30 \%$ aller Neurentner aus.

(3) Zur dritten Konstellation zählen Personen, die in den letzten drei Jahren in einen „prekären“ Status wechselten und aus ihm heraus in Altersrente gingen, ohne die ganze Zeit über arbeitslos, krank oder geringfügig beschäftigt gewesen zu sein, wie es für die zweite Konstellation gilt. Dieser erwerbsbiografische Typ lässt sich als „,kurzer Altersübergang" bezeichnen. Die schwache Arbeitsmarktbeteiligung kurz vor Rentenbeginn dauerte nicht lange an; möglicherweise wurde sie mit Blick auf die nahe Rente begonnen oder akzeptiert. Immerhin 11,8 \% der Neuzugänge gehören dieser Gruppe an.

Abb. 7: Vorzeitiger Altersrentenzugang von aktiv Versicherten nach typisierten Erwerbsbiografien vor Rentenbeginn, 2005 - in \% -

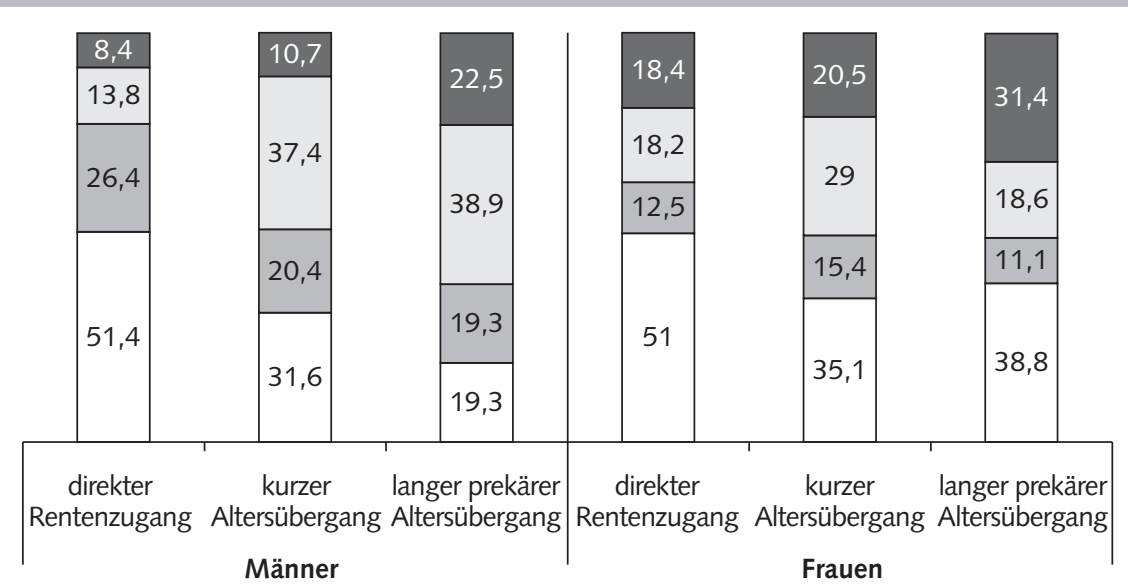

Vorzeitiger Rentenzugang um

$\square 60$ Monate $\square 25$ - 59 Monate $\square 1$ - 24 Monate $\square$ keine Abschläge

Quelle: FDZ-RV SUFRTZNO5XVSBB, zitiert nach Brussig 2007

WSI

Ostdeutsche sind hier anteilig mehr als doppelt so häufig vertreten $(24,1 \%)$.

Abbildung 7 stellt für Männer und Frauen getrennt dar, wie sich die vorzeitigen Rentenzugänge je nach der Erwerbsbiografie kurz vor Rentenbeginn unterscheiden.

Bei den Männern zeigen sich erhebliche Unterschiede je nach erwerbsbiografischer Konstellation (Abbildung 7). Einen abschlagsfreien Rentenzugang erreichten $51,4 \%$ der Männer, die in den drei Jahren vor Rentenbeginn dauerhaft erwerbstätig waren, aber nur 19,3 \% der Männer, die im selben Zeitraum dauerarbeitslos o.ä. waren. Ähnlich ausgeprägt ist der Unterschied beim frühestmöglichen Rentenzugang mit 60 Jahren (oberstes Segment jeder Säule in Abbildung 7): Mehr als jeder Fünfte der langzeitarbeitslosen bzw. durchgängig geringfügig beschäftigten Männer (22,5 \%) geht mit maximalen Rentenabschlägen in Höhe von $18 \%$ in Rente, aber nur 8,4 \% derjenigen, die vor Rentenbeginn drei Jahre durchgängig beschäftigt waren. ${ }^{10}$ Deutlich über die Hälfte der Personen mit langem prekären Altersübergang geht mehr als zwei Jahre vorgezogen in Rente (61,4 \%).

Frauen gehen häufiger als Männer zum frühestmöglichen Zeitpunkt in Rente. Aber ähnlich wie bei den Männern zeigt sich auch bei Frauen, dass sie öfter zum frühestmöglichen Zeitpunkt bzw. mit höheren Abschlägen ihre Rente beginnen, wenn sie kurzzeitig oder dauerhaft arbeitslos waren, ein prekäres Beschäftigungsverhältnis hat- ten oder ähnliches. Dies ist ein Hinweis darauf, dass auch bei Frauen eine feste Erwerbsintegration die Wahrscheinlichkeit eines abschlagsfreien Rentenzugangs erhöht. Beim Rentenübergang langzeitarbeitsloser Frauen zeichnet sich zudem eine Polarisierung ab. Ungefähr die Hälfte der Frauen im langen prekären Altersübergang geht nur mit geringen (bis 24 Monate) oder gar keinen Abschlägen in Ruhestand. Diese Frauen können - möglicherweise gestützt durch die fortdauernde Erwerbstätigkeit ihrer Ehemänner - auf das Erreichen einer abschlagsfreien Altersgrenze ,warten“.

Die durchschnittlichen Rentenzahlbeträge betragen 2005, bezogen auf alle drei erwerbsbiografischen Konstellationen, ca. $966 €$ (Männer) bzw. $587 €$ (Frauen). Sie liegen deutlich über den Durchschnittsrenten aller Neurentnerinnen und -rentner des Jahres 2005 (802€/463€), da in den hier betrachteten erwerbsbiografischen Konstellationen vor Rentenbeginn nur aktiv

\footnotetext{
Hinter dem Status "Anrechnungszeit" verbergen sich viele kranke - nicht erwerbstätige - Menschen sowie arbeitslose Personen ohne Leistungsbezug (Himmelreicher 2006).

9 Leistungsbezug nach dem SGB II am 13.12. des Vorjahres kann bei den hier betrachteten Rentenzugängen des Jahres 2005 noch nicht vorkommen.

10 Hinzuzurechnen sind jene Personen, die einer Schwerbehindertenrente zugehen, die ebenfalls frühestens $a b 60$ Jahre zugänglich ist, aber aufgrund der Altersgrenze von 63 Jahren maximal 36 Abschlagsmonate bzw. 10,8 \% Abschläge hinnehmen.
} 


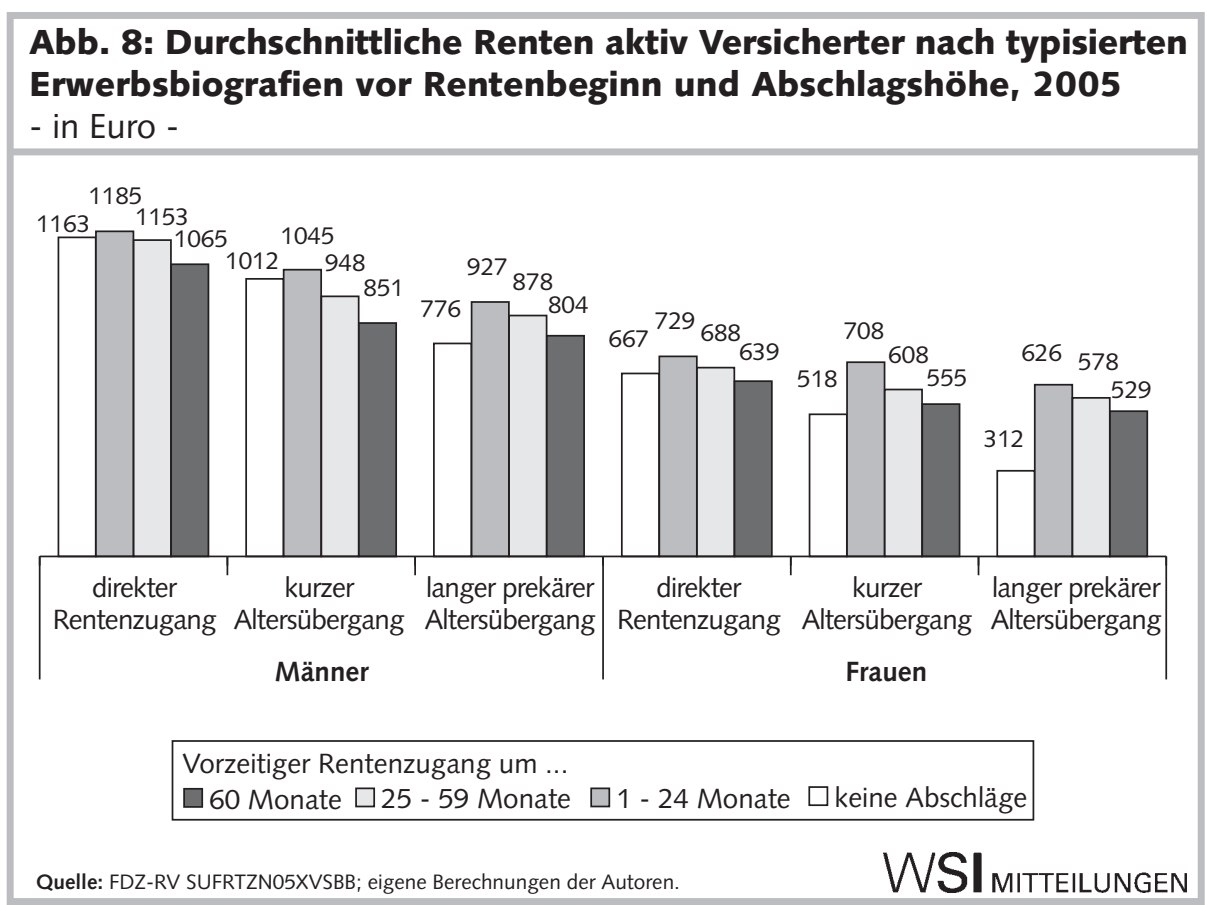

Versicherte enthalten und Nichterwerbstätige, Selbstständige und Beamte mit typischerweise geringen Ansprüchen in der Gesetzlichen Rentenversicherung nicht vertreten sind.

Vor allem bei Männern im langen prekären Altersübergang (Dauerarbeitslosigkeit, geringfügige Beschäftigung oder Krankheit in den letzten drei Jahren vor Rentenbeginn) sind die vorgezogenen Rentenzugänge mit unterdurchschnittlichen Rentenzahlbeträgen verbunden; bei einem um mindestens zwei Jahre vorgezogenen Rentenzugang liegt die Durchschnittsrente bei etwa $880 €$. Bei einem mit maximalen Abschlägen verbundenen Rentenzugang betragen die Durchschnittsrenten sogar nur ca. $800 €$. Die Kombination von vorzeitigem Rentenzugang (um mindestens zwei Jahre) und Arbeitslosigkeit o. ä. vor Rentenbeginn („langer“ oder „kurzer“ prekärer Altersübergang) führt stets zu unterdurchschnittlichen Rentenzahlbeträgen. Bei Frauen sind die Zusammenhänge zwischen dem Rentenzahlbetrag, dem Zeitpunkt des Eintritts in Rente und der erwerbsbiografischen Konstellation vor Rentenbeginn weniger eindeutig. Doch nicht anders als bei den Männern erhalten jene Frauen im Durchschnitt die höchsten Renten, die unmittelbar bis zum Rentenbeginn oder bis kurz davor erwerbstätig waren. Diese Differenzierung ist teilweise eine Folge der in Kauf genommenen Abschläge und der durch die Form des Altersübergangs in den letzten Jahren vor Rentenbe-

In den letzten zehn Jahren haben sich die Bedingungen der Alterserwerbstätigkeit und des Rentenzugangs ebenso deutlich verändert wie die Erwerbsbeteiligung und das Verhalten beim Rentenbeginn. Weitere
Modifikationen, die in den nächsten fünf Jahren wirksam werden, sind bereits beschlossen. Beispielhaft zu nennen sind die Schließung des erleichterten Bezugs von Leistungen der Arbeitslosenversicherung für Neuzugänge ab 2008 sowie der Wegfall der Förderung der Altersteilzeit durch die BA für Neuzugänge ab 2010. Hierüber konnten sich Betriebe unter bestimmten Bedingungen Personalzusatzkosten im Zusammenhang mit der Altersteilzeitbeschäftigung erstatten lassen. Hinzu kommen die Schließung einiger vorzeitig beziehbarer Altersrenten für Personen, die nach 1951 geboren wurden ${ }^{11}$ sowie die Anhebung der Altersgrenze für die Regelaltersrente $a b$ 2012.

In der jüngsten Vergangenheit waren zudem einige Trendbrüche zu beobachten. $\mathrm{Zu}$ erinnern ist an

- einen zuletzt wieder zunehmenden Anteil von Personen, die mit 60 Jahren in Altersrente gehen;

- einen leicht ansteigenden Anteil von Rentenzugängen über die Altersrente wegen Arbeitslosigkeit;

- rückläufige Zugänge in Altersrente aus unmittelbar vorhergehender versicherungspflichtiger Beschäftigung (einschließlich Altersteilzeitarbeit) sowie

- die zunehmende Beschäftigungslosigkeit Älterer bei gleichzeitig steigender Alterserwerbsbeteiligung.

Ungewiss ist, ob es sich hierbei tatsächlich um "Wendepunkte" handelt, die zumindest für Teilgruppen eine erneut zunehmende Kluft zwischen Erwerbsaustritt und Renteneintritt anzeigen, oder ob sich dahinter eher demografische Effekte verbergen, die nur vorübergehend die Bilanz eintrüben. Darüber hinaus ist von Interesse, ob die Rentenzugänge mit Abschlägen auch dann noch zunehmen, wenn die Abschläge in beinahe allen Rentenarten ihre maximale Höhe erreicht haben. Das alles spricht dafür, das Monitoring des Altersübergangs fortzusetzen. Nur so können bisherige Entwicklungen weiter verfolgt wie auch die künftigen Auswirkungen bereits beschlossener, aber noch nicht wirksamer Reformen beobachtet und analysiert werden.
11 Es laufen die „Arbeitsmarktrenten“ aus (Alters- rente wegen Arbeitslosigkeit und nach Altersteil- zeit sowie die Altersrente für Frauen). Weiterhin wird es die Altersrente für Schwerbehinderte und die Altersrente für langjährig Versicherte geben.


Bellmann, L./Gewiese, T./Leber, U. (2006): Betriebliche Altersstrukturen in Deutschland, in: WSI-Mitteilungen 8, S. 427-432

Börsch-Supan, A. (2004): Faire Abschläge in der gesetzlichen Rentenversicherung, in: Sozialer Fortschritt 10, S. 258-261

Bosch, G./Schief, S. (2005): Politik für ältere Beschäftigte oder Politik für alle? Zur Teilnahme älterer Personen am Erwerbsleben in Europa. IAT-

Report 04, Gelsenkirchen

Brussig, M. (2007): Vier von zehn Zugängen in Altersrente erfolgen mit Abschlägen - Massive Einbußen beim Rentenanspruch durch vorzeitigen Renteneintritt bei langzeitarbeitslosen Männern. IAQ(Altersübergangs)Report 01, Gelsenkirchen, online: http://www.iaq.uni-due.de/auemreport/2007/auem2007-01.shtml.

Brussig, M./Wübbeke, Ch. (2008): Policy-making in ageing labour markets: the case of hidden early retirement in Germany, in: Kuhn, M./Ochsen, C. (Hrsg.): European Labour Markets and Demographic Change, im Erscheinen

Brussig, M./Knuth, M. (2006): Altersgrenzenpolitik und Arbeitsmarkt. Zur Heraufsetzung des gesetzlichen Rentenalters, in: WSI-Mitteilungen 6, S. 307-313

Brussig, M./Wojtkowski, S. (2006): Durchschnittliches Renteneintrittsalter steigt weiter. Wachsende Differenzierung im Rentenzugangsalter seit 2003 zu beobachten. IAQ(Altersübergangs)-Report 02, Gelsenkirchen, online: http://www.iaq.uni-due.de/auem-report/2006/2006-02/ auem2006-02.pdf.

Brussig, M./Wojtkowski, S. (2007a): Rückläufige Zugänge in Altersrenten aus sozialversicherungspflichtiger Beschäftigung - steigende Zugänge aus Arbeitslosigkeit. IAQ(Altersübergangs)-Report 02, Gelsenkirchen, online: http://www.iaq.uni-due.de/auem-report/2007/auem2007-02. shtml.

Brussig, M./Wojtkowski, S. (2007b): Mehr Ältere auf dem Arbeitsmarkt: Erwerbstätigkeit und Arbeitslosigkeit nehmen zu. IAQ(Altersübergangs)Report 03, Gelsenkirchen, online: http://www.iaq.uni-due.de/auemreport/2007/auem2007-03.shtml.

Brussig, M./Wojtkowski, S. (2008): Anstieg der Alterserwerbsbeteiligung: Aktuelle demographische Veränderungen geben Rückenwind. IAQ(Altersübergangs)-Report 01, online: http://www.iaq.uni-due.de/ auem-report/2008/auem2008-01.shtml.

Bundesagentur für Arbeit (BA) (2007): Arbeitsmarktberichterstattung. Situation von Älteren auf dem Arbeitsmarkt - Erwerbstätigkeit, Beschäftigung und Arbeitslosigkeit, Stand Oktober

Büttner, R. /Knuth, M. (2004): Spätere Zugänge in Frührenten - Regelaltersrente auf dem Vormarsch. Verschiebung der Altersgrenzen und Abschlagsregelungen bewirken Verhaltensänderung der Versicherten. IAQ(Altersübergangs)-Report 01, online: http://www.iaq.uni-due.de/ auem-report/2004/2004-01/auem2004-01.pdf.
Deutsche Rentenversicherung Bund (DRV) (2007): Rentenversicherung in Zeitreihen, DRV-Schriften 22, Berlin

Deutscher Bundestag (2007): Bundestags-Drucksache 16/7447. Antwort der Bundesregierung. Ursachen für den statistisch festgestellten Anstieg der Beschäftigungsquote Älterer

Ebert, A./Fuchs, T./Kistler, E. (2006): Arbeiten bis 65 oder gar bis 67? Die Voraussetzungen fehlen, in: WSI-Mitteilungen 9, S. 492-499

Eichhorst, W. (2006): Beschäftigung Älterer in Deutschland: Der unvollständige Paradigmenwechsel, IZA discussion paper 1985, Bonn

Eichhorst, W. (2008): Von der Frühverrentung zum längeren Erwerbsleben: Transferleistungen, Arbeitsmarktpolitik und Weiterbildung, in: Sozialer Fortschritt 2, S. 24-34

Eichhorst, W./Sproß, C. (2005): Arbeitsmarktpolitik für Ältere: Die Weichen führen noch nicht in die gewünschte Richtung, IAB-Kurzbericht 16, Nürnberg

Europäische Union (EU) (2001): Schlussfolgerungen des Vorsitzes. Europäischer Rat (Stockholm), 23. und 24. März 2001, online http://www.consilium.europa.eu/ueDocs/cms Data/docs/pressData/ de/ec/ACF191B.html

Europäische Union (EU) (2005): Entscheidung des Rates vom 12. Juli über Leitlinien für beschäftigungspolitische Maßnahmen der Mitgliedstaaten, online: http://ec.europa.eu/employment_social/employment strategy/guidelines_de.htm

Himmelreicher, R. K. (2006): Analysepotenzial des Scientific Use File Versichertenrentenzugang, in: DRV Bund (Hrsg.): Forschungsrelevante Daten der Rentenversicherung. Bericht vom zweiten Workshop des Forschungsdatenzentrums der Rentenversicherung (FDZ-RV) vom 27. bis 29. Juni in Würzburg, DRV-Schriften 55, Berlin, S. 38-92

Hoffmann, H. (2007): Wege in den Ruhestand, in: Deutsche Rentenversicherung 4/5, S. 298-320

Kaldybajewa, K. (2004): Rentenzugang BfA 2003: Jeder achte Altersrentner kommt aus der Altersteilzeit, in: Die Angestellten-Versicherung 5/6, S. 227-236

Kistler, E./Ebert,A./Stecker, Ch. (2007): Steigende Beschäftigung Älterer: Sind wir wirklich auf dem richtigen Weg?, in: Deutsche Rentenversicherung 10, S. 651-664

Ohsmann, S./Stolz, U./Thiede, R. (2004): Rentenabschläge bei vorzeitigem Rentenbeginn: Was ist versicherungsmathematisch fair?, in: Sozialer Fortschritt 10, S. 267-271

Pimpertz, J. (2004): Wie hoch sollten die Rentenabschläge bemessen sein?, in: Sozialer Fortschritt 10, S. 262-267 